\title{
Treatment of hepatic pancreatic ductal adenocarcinoma metastases with high-dose-rate image-guided interstitial brachytherapy: a single center experience
}

\author{
Ralph Drewes, MD!', Jazan Omari, MD', Matthias Manig, MD', Max Seidensticker, MD², Peter Hass, MD', Jens Ricke, MD², \\ Maciej Powerski, MD!, Maciej Pech, MD',3 \\ 'Department of Radiology and Nuclear Medicine, Otto-von-Guericke University, Magdeburg, Germany, ${ }^{2}$ Clinic and Department of \\ Radiology, Munich University, Munich, Germany, ${ }^{3} 2^{\text {nd }}$ Department of Radiology, Medical University of Gdansk, Gdansk, Poland
}

\begin{abstract}
Purpose: To evaluate the efficacy and safety of image-guided (computed tomography/magnetic resonance imaging - CT/MRI) high-dose-rate (HDR) interstitial brachytherapy (iBT) as a salvage maneuver for the treatment of hepatic metastases originating from hepatic pancreatic ductal adenocarcinoma (PDAC). PDAC metastases present a major and unresolved problem, and any surgical approach or local therapeutic intervention remains extremely controversial.

Material and methods: A cumulative number of 45 hepatic PDAC metastases in 16 patients were treated and retrospectively analyzed. Synchronous metastatic spread was observed in five patients, metachronous in eleven. 14 patients had resection of the pancreatic primary prior to iBT: eight Whipple/PPPD and six distal pancreatectomy procedures. The hepatic metastases were progressing under chemotherapy, thus iBT was applied as a salvage maneuver with the intention of local tumor control and prolonged survival. iBT is applied interstitially, with temporarily introduced ${ }^{192} \mathrm{Ir}$ source in a single fraction HDR irradiation regime to eradicate vital tumor cells. Response to treatment was assessed clinically with CT/MRI every three months.

Results: Local tumor control was achieved in $87 \%$ of all treated metastases. The median diameter of the irradiated lesions was $2.2 \mathrm{~cm}$ (range, 1-11.2 cm), the median irradiation dose was 21 Gy (range, 5-29.1 Gy). Median progression-free survival (PFS) after iBT was 3.4 months (range, 1.5-19.6 months), the median overall survival (OS) after iBT was 8.9 months (range, 3.1-29.3 months). Three major complications (CTCAE grade 3) occurred following iBT: three cases of liver abscess, which were successfully resolved with drainage and antibiotics.

Conclusions: Overall, iBT is a safe procedure, which enables excellent rates of local tumor control and presents a viable anti-neoplastic treatment option as a salvage therapy for metastatic PDAC patients.

J Contemp Brachytherapy 2019; 11, 4: 329-336

DOI: https://doi.org/10.5114/jcb.2019.87269
\end{abstract}

Key words: PDAC, interstitial brachytherapy, local ablation, local tumor control, salvage.

\section{Purpose}

Pancreatic cancer is the fourth most fatal cancer in both women and men, with a life expectancy of 2-5\% at 5 years $[1,2]$. Moreover, most patients have already progressed to an advanced or metastatic stage of the disease at the time of diagnosis. Several preclinical studies established that pancreatic ductal adenocarcinoma (PDAC) is a systemic disease from the outset, displaying early micrometastatic spread [3,4]. Autopsy studies of primary resected PDAC patients showed that $70-85 \%$ of patients die of systemic recurrence rather than local disease [5].
Only $10-15 \%$ of all patients are eligible for surgery, which is presently considered to be the only potentially curative approach [6,7]. Even after surgery, PDAC remains highly lethal, as many patients develop hepatic metastases. Resectability status mainly depends on peripancreatic vessel contact/infiltration and presence or absence of distant metastases [8]. However, many PDAC patients undergo surgery of the primary tumor at some point and consequently have a biliodigestive anastomosis (BDA), which ultimately results in bacterial colonization of the intrahepatic bile ducts and complicating any hepatic metastasis treatment [9].
Address for correspondence: Ralph Drewes, MD, Department of Radiology and Nuclear Medicine, Otto-von-Guericke University, 44 Leipziger St., 39120 Magdeburg, Germany, phone: +49 3916713030 , fax: +49391 6713029, 凶e-mail: ralph.drewes@med.ovgu.de
Received: 20.02 .2019

Accepted: 23.07.2019

Published: 29.08.2019 
Treatment of PDAC metastases is challenging, since partial hepatectomy as a principal method not only failed to show any promising results, but also cannot usually be performed repeatedly due to impairment of liver function and the patient's general condition [10,11]. Alternative measures like thermal liver ablation (RFA, laser therapy), are often complicated by cholestasis, bile duct strictures, and hepatic abscesses, with even higher incidence rates after previously performed BDA [12]. Even though being minimal invasive, thermal ablation measures underlie several restrictions such as tumor size $(<5 \mathrm{~cm})$, heat sink effect, and inability to be used near thermosensitive structures.

In contrast to the aforementioned therapies, imageguided high-dose-rate brachytherapy (iBT) presents a different, anti-neoplastic, transcutaneous, and minimally invasive treatment option, and is applied in this study. Its efficacy and ability to provide local tumor control (LTC) has been proven by several investigators for different tumor entities in the past, achieving excellent local tumor control rates around 90\% $[13,14,15,16]$. iBT is an afterloading technique that employs a ${ }^{192}$ Ir source, which is placed temporarily into the clinical target volume, i.e. the tumor. High-dose-rate irradiation is applied in a single fraction, providing an extensive cytotoxic effect via DNA and RNA damage to eradicate vital tumor cells. Other researchers examined the use of iBT for the treatment of patients with PDAC liver metastases and demonstrated a high local tumor control rate of 91\% [17].

The goal of our study was to assess the efficacy and safety of iBT as a salvage maneuver for the treatment of liver metastases originating from PDAC.

\section{Material and methods \\ Patient characteristics}

Sixteen patients, with histologically proven PDACs and a cumulative number of 45 unresectable liver metastases, received treatment with iBT in our department between February 2010 and March 2017, and were enrolled in this retrospective study. Every patient was in a metastatic and progressive stage of disease at the time of referral to our department. Our study was approved by the local ethics committee.

\section{Study design and eligibility criteria}

Local tumor control (LTC) and overall safety of iBT were the primary endpoints of this retrospective study.

Each individual PDAC patient's case was discussed at an interdisciplinary board of oncologists, interventional radiologists, radiation oncologists, and visceral surgeons who determined the indication for iBT for each patient individually.

The inclusion criteria were: 1) Resection impossible or unfavorable due to perioperative risk or loss of liver function; 2) Patient unwilling to undergo surgery, 3) Oligometastatic ( $\leq 5$ metastases upon initial presentation)/ controllable disease extent; 4) Adequate coagulation parameters (thrombocytes $>50000 / \mathrm{nl}$, prothrombin $>50 \%$, partial thromboplastin time $<50 \mathrm{~s}$ ). Exclusion criteria were correspondingly: 1) Lack of consent, and 2) Uncontrollable tumor spread.

\section{Interventional technique and irradiation}

Prior to the iBT procedure, a whole-body contrast-enhanced CT and a Gb-EOB-DTPA-enhanced liver MRI (Primovist, Bayer Pharma, Leverkusen, Germany) were acquired for treatment planning and staging purposes. Physical status and laboratory parameters were also evaluated.

During and prior to the intervention, analgesia (fentanyl), sedation (midazolam), and local anesthesia (lidocaine) were administered. An 18-gauge needle was used under CT fluoroscopic guidance (Toshiba, Aquilion, Japan) or real time 1.0 Tesla MRI (Panorama 1.0T, open MR system, Philips Healthcare) to puncture the target lesions. In a next step, a flexible 6-French catheter sheath (Radifocus, Terumo ${ }^{\mathrm{TM}}$ ) was placed using Seldinger technique over a stiff angiography guidewire (Amplatz, Boston Scientific, Marlborough, USA). Finally, the 6-French afterloading catheter (Afterloadingkatheter, Primed Medizintechnik Gmbh, Halberstadt, Germany) was introduced and the catheter ending temporarily fixated to the skin with sterile bandages and a cutaneous suture. Target lesion size and nearby structures at risk determined the number of catheters and their angulation. A CT scan in breath-holding technique or a Gadolinium-enhanced MRI were acquired for further treatment and irradiation planning as well as for catheter positioning confirmation. The interventional radiologist and the radiotherapist marked the clinical target volume and the adjacent organs at risk in every CT or MRI slice.

The irradiation design was devised employing the acquired dataset and the software system Oncentra (Nucletron, Elekta Ab, Stockholm, Sweden). The software was a part of the HDR afterloading system. The three-dimensional coordinates $(x, y, z)$ of each positioned catheter's tip in relation to the tumor margins were transferred into the treatment planning system. Furthermore, the calculated isodose lines were inspected in every imaging slice and adapted to the target lesion margins. An imaging example of the interventional technique is illustrated in Figure 1.

The afterloading/iBT system (Nucletron, Elekta Ab, Stockholm, Sweden) applied an ${ }^{192}$ Ir source with a nominal activity of $10 \mathrm{Ci}(370 \mathrm{GBq})$. The irradiation was administered in a single fraction. The reference dose was defined as 20 Gy to enclose the entire target lesion (D99.9\%); even higher, exponentially increasing doses were applied at the target lesion's irradiation center. Prevention of new peripheral tumor incidences was achieved through implementation of a 5-millimeter security margin around the target lesion, i.e., the clinical target volume (CTV). Adjacent organs at risk such as the gastrointestinal tract (GI) were respected, and the irradiation scheme and dose correspondingly adjusted (empiric GI tract dose < 14 Gy/ml) [18].

Upon completion of the iBT procedure, the catheters were removed. The puncture sites were sealed by injection of gelfoam or fibrin tissue glue. 

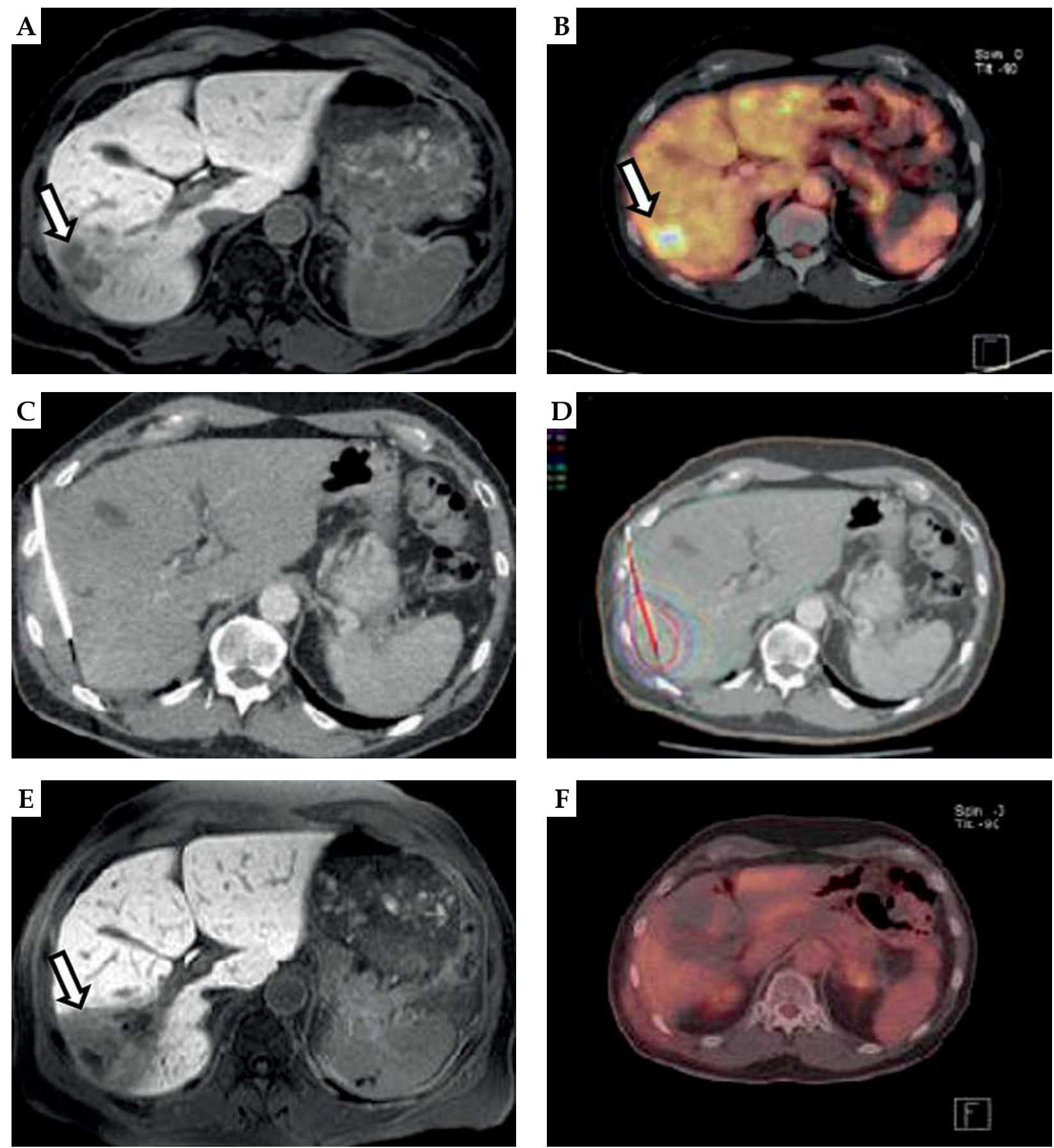

Fig. 1. Local tumor control in a patient with metastatic PDAC. A) Axial T1w Gd-EOB-DTPA (Primovist)-enhanced MRI (baseline MRI prior to iBT), arrow points to liver metastases; B) FDG-PET-CT demonstrates the activity of the hepatic lesions (arrow) prior to iBT; C) Inserted brachytherapy catheter in the liver lesions (white arrow) during CT-guided iBT; D) Colored lines represent the irradiation isodoses, with red line showing 20 Gy; E) Axial T1w Gd-EOB-DTPA-enhanced follow-up MRI after iBT with Gd-EOB-DPTA enhancement defect following irradiation; F) FDG-PET-CT (follow-up) shows no activity in the hepatic ablation area

\section{Follow-up}

Response to iBT treatment was evaluated every three months after the ablation procedure: a Gb-EOB-DTPAenhanced liver MRI, a contrast-enhanced CT (thorax and abdomen), clinical and laboratory evaluations were performed. Changes in size and enhancement defects were correlated in a dynamic T1w GRE sequence, DWI/ADC, post-Gd-EOB-DTPA, and a T2w sequence. Tumor edema was visualized in a $\mathrm{T} 2 \mathrm{w}$ sequence, vital tumor tissue in DWI and late enhancement (post-radiation) defects in the post-Gd-EOB-DTPA sequence and the contrast agent dynamic sequences. Measurements were ultimately made in axial slices of the post-Gd-EOB-DTPA sequence in cor- 
relation with the DWI to account for vital tumor tissue and to differentiate from late enhancement defects. In some cases, an FDG-PET-CT was acquired.

Adverse events were recorded and defined corresponding to the Common Terminology for Adverse Events (CTCAE), version 4.03.

Local tumor control (LTC) after brachytherapy was defined corresponding to the Response Evaluation Criteria in Solid Tumors (RECIST 1.1) categories as stable disease (SD), partial remission (PR), and complete remission (CR). Progressive disease was defined as an increase of tumor diameter $>20 \%$ during follow-up.

\section{Statistical methods}

The primary objectives of this retrospective, single arm study were local tumor control and the overall safety of iBT. Progression-free survival and overall survival

Table 1. Patients characteristics

\begin{tabular}{|c|c|}
\hline Total number of patients & 16 \\
\hline \multicolumn{2}{|l|}{ Sex } \\
\hline Men & 10 \\
\hline Women & 6 \\
\hline \multicolumn{2}{|l|}{ Age at time of diagnosis } \\
\hline Median & $62\left(Q_{1}=55, Q_{3}=69\right)^{1}$ \\
\hline Range & $35-73$ \\
\hline Primary localization & 16 \\
\hline Caput (head) & 9 \\
\hline Cauda (tail) & 6 \\
\hline Corpus (body) & 1 \\
\hline Chemotherapy (before iBT) ${ }^{2}$ & 16 \\
\hline Resection of the primary (before iBT) & $14 / 16$ \\
\hline Whipple \& PPPD & 8 \\
\hline Distal pancreatectomy & 6 \\
\hline \multicolumn{2}{|l|}{ Other therapies } \\
\hline Partial hepatectomy \& radiation & 1 \\
\hline SIRT & 1 \\
\hline IBT primary (no resection) & 1 \\
\hline ERCP (caput primary) & 3 \\
\hline Metastases (cumulative) & 45 \\
\hline Liver & 45 \\
\hline \multicolumn{2}{|l|}{ Type of metastatic spread } \\
\hline Synchronous & 5 \\
\hline Metachronous & 11 \\
\hline \multicolumn{2}{|l|}{ Lesion size (max diameter in $\mathrm{cm}$ ) } \\
\hline Median & $2.2\left(\mathrm{Q}_{1}=1.3, \mathrm{Q}_{3}=3.3\right)$ \\
\hline Range & $1-11.2$ \\
\hline \multicolumn{2}{|l|}{ Irradiation dose (iBT) (Gy) } \\
\hline Median & $21\left(\mathrm{Q}_{1}=17, \mathrm{Q}_{3}=24\right)$ \\
\hline Range & $5-29.1$ \\
\hline
\end{tabular}

were secondary objectives. Calculations of LTC, PFS, and OS were done using Kaplan-Meier method with SPSS version 22 (SPSS, version 22.0; SPSS, Chicago Illinois).

\section{Results}

Sixteen patients with histologically proven PDAC, having a cumulative overall amount of 45 liver metastases, were treated with iBT in our department between 2010 and 2017, and were included in this retrospective study (Table 1). The median patient age at the time of diagnosis was 62 years (range, 35-73 years). Localization of the pancreatic primary tumor was as follows: nine in the head, six in the tail, and one in the body. Fourteen patients had a resection of the primary tumor prior to iBT: eight cases of Whipple/PPPD procedure and six cases of distal pancreatectomy. One patient's primary was treated with iBT instead of surgery. Only one patient neither had resection nor iBT of the primary.

\begin{tabular}{|c|c|}
\hline \multicolumn{2}{|l|}{ Irradiation time (iBT) (min) } \\
\hline Median & $29.8\left(Q_{1}=13.7, Q_{3}=38.3\right)$ \\
\hline Range & $8-82.8$ \\
\hline \multicolumn{2}{|l|}{ Number of catheters/lesion } \\
\hline Median & $1\left(\mathrm{Q}_{1}=1, \mathrm{Q}_{3}=2\right)$ \\
\hline Range & $1-6$ \\
\hline Local tumor control & $39 / 45(86.7 \%)$ \\
\hline \multicolumn{2}{|l|}{ Local tumor control time (months) } \\
\hline Median & $3.3\left(\mathrm{Q}_{1}=2.8, \mathrm{Q}_{3}=5.5\right)$ \\
\hline Range & $1.5-27.9$ \\
\hline \multicolumn{2}{|l|}{ Progression-free survival (months) } \\
\hline Median & $3.4\left(\mathrm{Q}_{1}=2.8, \mathrm{Q}_{3}=6.5\right)$ \\
\hline Range & $1.5-19.6$ \\
\hline \multicolumn{2}{|l|}{ Overall survival after iBT (months) } \\
\hline Median & $8.9\left(\mathrm{Q}_{1}=5.6, \mathrm{Q}_{3}=8.9\right)$ \\
\hline Range & $3.1-29.3$ \\
\hline \multicolumn{2}{|l|}{ OS from time of diagnosis (months) } \\
\hline Median & $27.5\left(\mathrm{Q}_{1}=19.5, \mathrm{Q}_{3}=51.3\right)$ \\
\hline Range & $13-63$ \\
\hline \multicolumn{2}{|l|}{ Previous treatment (before iBT) } \\
\hline Chemotherapy & $16(100 \%)$ \\
\hline Resection & $14(87.5 \%)$ \\
\hline Selective internal radiotherapy & 1 \\
\hline IBT primary (no resection) & 1 \\
\hline \multicolumn{2}{|l|}{ IBT image guidance } \\
\hline $\mathrm{CT}$ & 26 \\
\hline MRI & 19 \\
\hline \multicolumn{2}{|l|}{ Time of hospitalization (days) } \\
\hline Median & 4 \\
\hline Range & $3-6$ \\
\hline
\end{tabular}

${ }^{1}$ quartile range, ${ }^{2}$ image-guided high-dose-rate interstitial brachytherapy

Whipple \& PPPD - whipple procedure and pylorus preserving pancreaticoduodenectomy, SIRT - selective internal radiotherapy (radioembolization), ERCP - endoscopic retrograde cholangiopancreatography 
Table 2. Organs at risk and tumor dose overview

\begin{tabular}{|c|c|c|c|c|c|}
\hline & $\begin{array}{c}\text { D1 cc } \\
\text { Median (range) }\end{array}$ & V ${ }_{5}$ Gy (\%) & $\mathrm{D}_{90}$ & $\mathrm{D}_{99.9}$ & $\mathrm{D}_{\text {mean }}$ \\
\hline Gastr $(n=7)$ & $8.1(4.4-16.4)$ & $x$ & $x$ & $x$ & $x$ \\
\hline Duod $(n=1)$ & $x$ & $x$ & $x$ & $x$ & $x$ \\
\hline Colo $(n=3)$ & $8(4.7-19.8)$ & $x$ & $x$ & $x$ & $x$ \\
\hline Kidn $(n=4)$ & $16(12.1-21.2)$ & $x$ & $x$ & $x$ & $x$ \\
\hline Heart $(n=4)$ & $13.4(1.9-18.1)$ & $x$ & $x$ & $x$ & $x$ \\
\hline Liver & $x$ & $20(1.5-70)$ & $x$ & $x$ & $x$ \\
\hline Tumor $(n=45)$ & $x$ & $x$ & $31.5(15.5-81)$ & $21.1(11.5-62)$ & $20(1.5-70)$ \\
\hline
\end{tabular}

Table 2 shows 5 Gy liver volume \%, the organs at risk (OARs) dose (Gy/ml), the tumor doses $D_{90}, D_{99}$, and $D_{\text {mean }}(G y)$ in median and range. A cumulative number of 26 brachytherapy interventions were performed. The $n=\ldots$ states the number of interventions were each organ was at risk, e.g. gastr $n=7-g a s t r i c$ organ at risk in 7 out of 26 interventions (in 19 interventions $D_{1 \text { cc }}$ of $0 \mathrm{~Gy} / \mathrm{ml}$ )

Synchronous metastatic spread was observed in five patients and metachronous spread in eleven patients.

Every patient received some form of palliative chemotherapy and showed disease progression prior to iBT: gemcitabine was administered to twelve patients and FOLFIRINOX to four patients. Gemcitabine monotherapy was amended in some cases: two cases of additional erlotinib, three cases of additional paclitaxel and two cases of additional oxaliplatin.

In the recent follow-up staging CT before referral to our department, every patient's PDAC disease was found to be progressive under palliative chemotherapy; hence, iBT was applied as a salvage maneuver and chemotherapy discontinued four weeks prior to the iBT procedure. Disease progression was the primary reason for chemotherapy cancellation and drug-related toxicity was the secondary reason. Some patients received repeated iBT treatments, either to split the treatment and irradiation burden into two or more sessions, or to treat newly developed metastases later. A more detailed overview of the performed iBT and the dose applied is presented in Table 2.

\section{Treatment characteristics}

The median tumor diameter was $2.2 \mathrm{~cm}$ (range, 1-11.2 $\mathrm{cm})$. The number of inserted catheters per lesion during iBT varied between one and six, with a median of one. $\mathrm{CT}$ guidance was used in 26 interventions, MRI guidance in 19. The minimal planned/anticipated tumor enclosing dose was 20 Gy (D99.9\%), which had to be adapted in some cases due to risk structures in proximity - a median irradiation dose of 21 Gy (range, 5-29.1 Gy) was administered. The median total irradiation time was 29.8 minutes (range, 8-82.8 minutes).

The intended tumor enclosing dose (D99.9\%) was reached in 35 of $45(77.7 \%)$ of all treated metastases. For the treatment of the other 10 lesions, the dose had to be adjusted due to risk structures in proximity.

The time of hospitalization ranged between three and six days, with a median of four days. Three patients developed a liver abscess (CTCAE grade 3 ) following an iBT session, which was successfully resolved with transcutaneous drainage and antibiotics, each without signif- icant hospitalization prolongation. Four other patients received prophylactic periinterventional antibiotics as a precaution due to pre-existing, considerable cholestasis; no sign of infection or liver abscess was observed.

\section{Local tumor control, overall survival, progression free survival}

Local tumor control was achieved in $87 \%$ of all treated lesions in the Kaplan-Meier analysis (Figure 2). A cumulative number of six local relapses (liver metastases) were observed in four patients; one patient had a relapse of every treated lesion (three in total). The median progression-free survival (PFS) was 3.4 months (Figure 3). The median overall survival of the 16 patients with metastatic PDAC, calculated from the time of iBT was 8.9 months (Figure 4). The median OS from the time of PDAC diagnosis was 27.5 months.

\section{Discussion}

PDAC mortality rates and OS remain poor and have barely improved in the last decades; median overall sur-

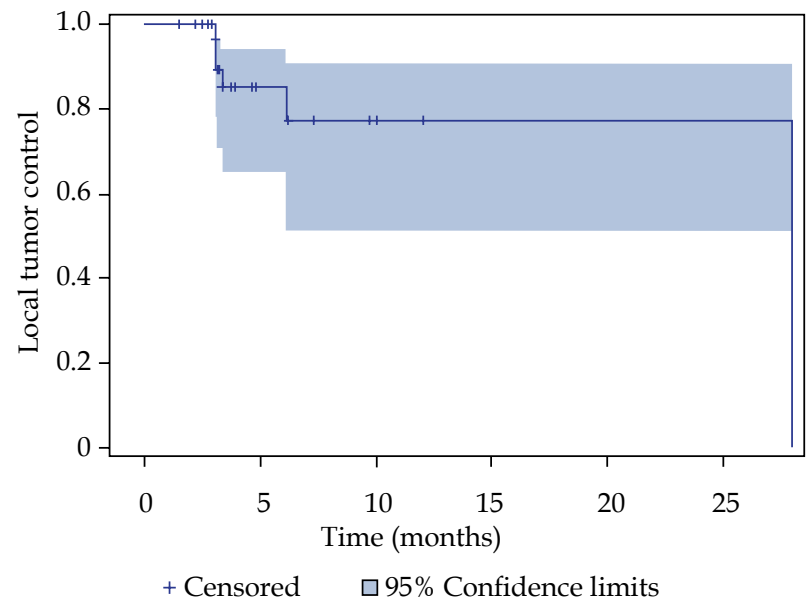

Fig. 2. Local tumor control (LTC) after iBT of pancreatic ductal adenocarcinoma (PDAC) metastases, estimated with the Kaplan Meier method 


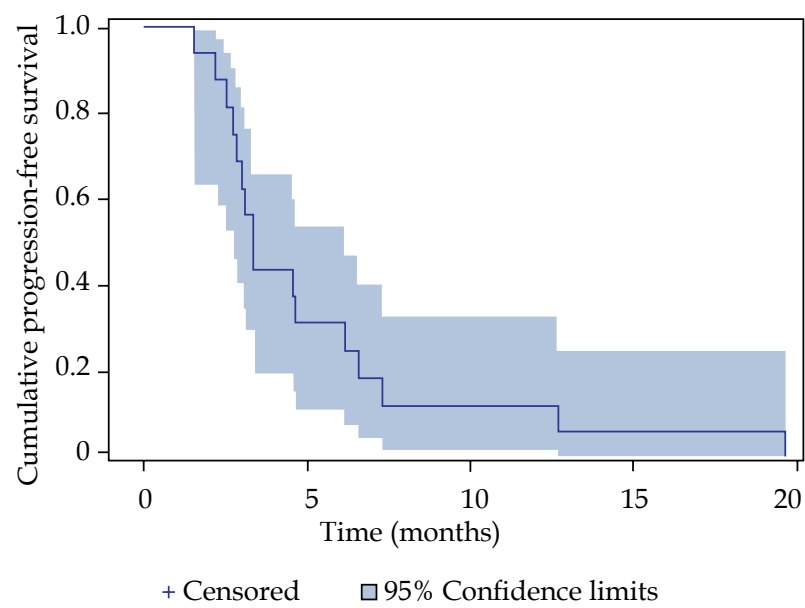

Fig. 3. Progression-free survival (PFS), calculated from the time of iBT, of patients with metastatic PDAC after treatment with iBT, estimated with the Kaplan Meier method

vival (mOS) across various studies is still less than two years, mOS of metastatic PDAC is about 6 months [19]. However, two RCTs established recent breakthroughs in first line chemotherapy with FOLFIRINOX (OS, 11.1 months) and the combination of gemcitabine and nab-paclitaxel (OS, 8.5 months) compared with the traditional gemcitabine monotherapy (OS, 6.8 months) [20,21]. The downside of these "new" first line regimens is their limited applicability. The administration is reasonable only to patients with an ECOG PS of 0 or 1 , which is the case for about $10-15 \%$ of all PDAC patients. In our study, four patients $(25 \%)$ initially had an adequate ECOG PS and received FOLFIRINOX. An ECOG PS of 2 limits the options to gemcitabine monotherapy according to guidelines, which was the case for other 12 patients in our study.

Results from randomized trials comparing chemotherapy with chemotherapy plus conventional external radiation indicate no significant survival improvement with additional radiation [22].

The current standard of care for early-stage disease is surgery followed by adjuvant chemotherapy. Although surgical resection is widely considered curative, observed outcomes across various studies fail to support that presumption. A large analysis of more than 300,000 patients from the National Cancer Database demonstrated that mOS after resection of the primary was only 13 months [23]. Other large RCTs and trials support that data: mOS was less than 2 years $[19,24,25]$. The 30 -day mortality after PDAC resection was up to $9 \%[19,24]$.

No treatment consensus exists regarding metastatic PDAC, which is considered unresectable based on the NCCN guidelines, apart from systemic therapy. While surgical metastasectomy being the standard method of choice for other cancer entities like colorectal cancer (CRC) or neuroendocrine liver metastases, the surgical approach fails to provide comparable promising results for PDAC metastases. The oncological value of liver surgery in PDAC patients is still highly questionable. Therefore, synchronous pancreatic and liver resections are only performed in very few PDAC cases, even in high-volume centers. A small

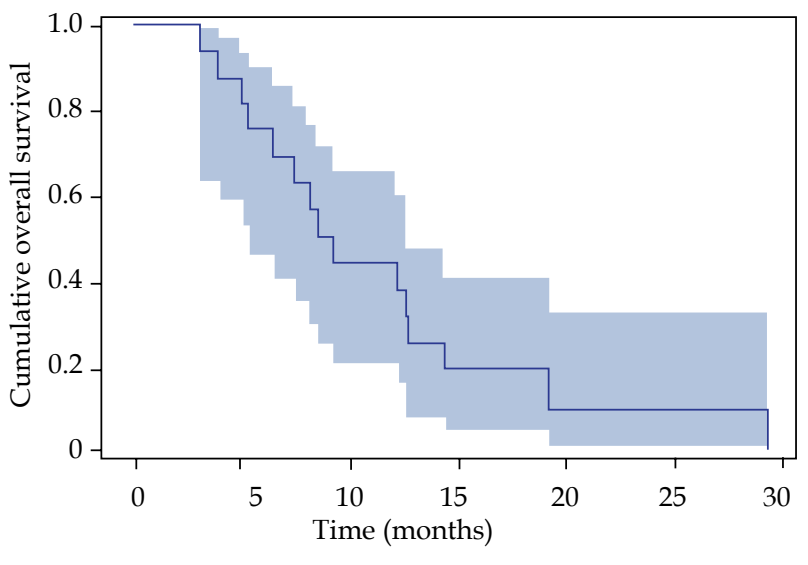

+ Censored $\quad \square 95 \%$ Confidence limits

Fig. 4. Overall survival (OS), calculated from the time of iBT, of patients with metastatic PDAC ablated with iBT, estimated with the Kaplan Meier method

study examined the outcome of seven patients after liver metastasectomy and published a mOS of 5.8 months [26]. Klempnauer et al. [26] reported a mOS of 8.3 months after synchronous liver and pancreatic resection and 5.8 months after metachronous hepatic resection. Klein et al. [27] published a mOS in a study $(n=22)$ of PDAC patients with synchronous hepatic metastasis resection of 7.6 months after surgery. Gleisner et al. [28] reported a mOS of 6 months even among highly selected patients with a low-volume metastatic liver disease. No benefit in overall survival was found in an older study by Takada et al. [29].

In contrast, the OS of our study calculated after iBT was 8.9 months, which is quite remarkable considering that all our patients had no further therapeutic options and were progressing under palliative chemotherapy, which was cancelled four weeks prior to iBT procedures. Furthermore, many of our patients had an unfavorable ECOG PS of 2, which rendered any surgical approach impossible. Re-challenge with alternative systemic anti-neoplastic regimens was either prohibited by the overall clinical condition or failed. To our knowledge, there is no comparable study evaluating the OS after resection of PDAC liver metastases in a salvage situation.

Another treatment approach for PDAC liver metastases are minimal invasive treatments like radiofrequency ablation. Park et al. came to the conclusion that selected patients with single, small sized $(<2 \mathrm{~cm})$ PDAC liver metastases gain a survival benefit through an application of RFA [30]. However, certain restrictions of thermal ablation methods like RFA limit its applicability; tumor size $<5 \mathrm{~cm}$, heat sink effect adjacent to vessels, high tumor vascularization, and proximity to central bile duct are the most important limitations.

In contrast, these restrictions do not apply to iBT, no size limit or cooling effects concerning brachytherapy are known. IBT even surpasses the size limit of $6 \mathrm{~cm}$ of stereotactic body radiation therapy (SBRT) and seems to induce fewer cases of radiation-induced liver disease (RILD) [31]. An advantage of local ablation measures like iBT or RFA concerning liver metastases is that it can be performed re- 
peatedly while preserving liver function. The issue of potential needle track metastasis was addressed specifically by radiation of the interventional access as a precaution.

The results of our study (mOS 8.9 months, PFS 3.4 months, LTC $87 \%$ ) confirm the data published by Wieners et al. (mOS 8.6 months, PFS 3.2 months, LTC 91\%) [17]. The observed complication rate is also similar; we report three major complications in 45 iBT procedures, whereas Wieners et al. also report three major complications in 49 iBT procedures - in both studies with hepatic abscesses. A crucial risk factor promoting liver abscess caused by ascending biliary infection based on bacterial colonization is a biliodigestive anastomosis (BDA) in patients with prior Whipple procedures. Correspondingly, the three cases with hepatic abscesses in our study, which were successfully resolved with drainage and antibiotics, also had a BDA following resection of the pancreatic head. According to literature, major adverse events (grade 3 and 4) after iBT are observed in about $3 \%$ of cases [32].

Despite the promising results, limitations of our study are the relatively small patient collective and the retrospective, single arm design. The outcome of our study, however, substantiates the findings of Wieners et al. suggesting that iBT might prolong OS in a metastatic setting, which generally implies dreadful prognosis. Further investigations in prospective RCTs are necessary to validate the results of our small, retrospective analysis. Until then, the current treatment rationale should be to identify eligible patients for local treatment options in combination with systemic chemotherapy to prolong survival.

\section{Conclusions}

Our study demonstrates and confirms that iBT is an overall safe procedure for the treatment of PDAC liver metastases and excellent local tumor control rates can be achieved.

\section{Disclosure}

Authors report no conflict of interest.

\section{References}

1. Sohal DP, Mangu PB, Khorana AA et al. Metastatic Pancreatic cancer: American Society of Clinical Oncology Clinical Practice Guideline. J Clin Oncol 2016; 34: 2784-2796.

2. Ducreux M, Cuhna AS, Caramella C et al. Cancer of the pancreas: ESMO Clinical Practice Guidelines for diagnosis, treatment and follow-up. Ann Oncol 2015; 26: v56-68.

3. Sohal DPS, Walsh RM, Ramanathan RK et al. Pancreatic adenocarcinoma: treating a systemic disease with systemic therapy. J Natl Cancer Inst 2014; 106: dju011.

4. Rhim AD, Mirek ET, Aiello NM et al. EMT and dissemination precede pancreatic tumor formation. Cell 2012; 148: 349-361.

5. Hishinuma S, Ogata Y, Tomikawa M et al. Patterns of recurrence after curative resection of pancreatic cancer, based on autopsy findings. J Gastrointest Surg 2006; 10: 511-518.

6. Riall TS, Cameron JL, Lillemoe KD et al. Resected periampullary adenocarcinoma: 5-year survivors and their 6- to 10-year follow-up. Surgery 2006; 140: 764-772.

7. Schnelldorfer T, Ware AL, Sarr MG et al. Long-term survival after pancreatoduodenectomy for pancreatic adenocarcinoma: is cure possible? Ann Surg 2008; 247: 456-462.
8. Tempero MA, Malafa MP, Al-Hawary M et al. Pancreatic adenocarcinoma, Version 2.2017, NCCN Clinical Practice Guidelines in Oncology. J Natl Compr Cancer Netw 2017; 15: 1028-1061.

9. De Jong MC, Farnell MB, Sclabas G et al. Liver-directed therapy for hepatic metastases in patients undergoing pancreaticoduodenectomy: a dual-center analysis. Ann Surg 2010; 252: 142-148.

10. de Jong MC, Tsai S, Cameron JL et al. Safety and efficacy of curative intent surgery for peri-ampullary liver metastasis. J Surg Oncol 2010; 102: 256-263.

11. Yamada H, Hirano S, Tanaka E et al. Surgical treatment of liver metastases from pancreatic cancer. HPB (Oxford) 2006; 8: 85-88.

12. Choi D, Lim HK, Kim MJ et al. Liver abscess after percutaneous radiofrequency ablation for hepatocellular carcinomas: frequency and risk factors. AJR Am J Roentgenol 2005; 184: 1860-1867.

13. Ricke J, Wust P, Wieners $G$ et al. Liver malignancies: CT-guided interstitial brachytherapy in patients with unfavorable lesions for thermal ablation. J Vasc Interv Radiol 2004; 15: 1279-1286.

14. Lüdemann $L$, Wybranski $C$, Seidensticker $M$ et al. In vivo assessment of catheter positioning accuracy and prolonged irradiation time on liver tolerance dose after single-fraction 192Ir high-dose-rate brachytherapy. Radiat Oncol 2011; 6: 107.

15. Tsalpatouros A, Baltas D, Kolotas C et al. CT-based software for 3-D localization and reconstruction in stepping source brachytherapy. IEEE Trans Inf Technol Biomed 1997; 1: 229242.

16. Collettini F, Poellinger A, Schnapauff D et al. CT-guided high-dose-rate brachytherapy of metachronous ovarian cancer metastasis to the liver: initial experience. Anticancer Res 2011; 31: 2597-2602.

17. Wieners G, Schippers AC, Collettini F et al. CT-guided highdose-rate brachytherapy in the interdisciplinary treatment of patients with liver metastases of pancreatic cancer. Hepatobiliary Pancreat Dis Int 2015; 14: 530-538.

18. Streitparth F, Pech M, Böhmig $M$ et al. In vivo assessment of the gastric mucosal tolerance dose after single fraction, small volume irradiation of liver malignancies by computed tomography-guided, high-dose-rate brachytherapy. Int $\mathrm{J} R a$ diat Oncol Biol Phys 2006; 65: 1479-1486.

19. Oettle H, Neuhaus P, Hochhaus A et al. Adjuvant chemotherapy with gemcitabine and long-term outcomes among patients with resected pancreatic cancer: the CONKO-001 randomized trial. JAMA 2013; 310: 1473-1481.

20. Von Hoff DD, Ervin T, Arena FP et al. Increased survival in pancreatic cancer with nab-paclitaxel plus gemcitabine. N Engl J Med 2013; 369: 1691-1703.

21. Conroy T, Desseigne F, Ychou M et al. FOLFIRINOX versus gemcitabine for metastatic pancreatic cancer. $N$ Engl J Med 2011; 364: 1817-1825.

22. Van Laethem JL, Hammel P, Mornex F et al. Adjuvant gemcitabine alone versus gemcitabine-based chemoradiotherapy after curative resection for pancreatic cancer: a randomized EORTC-40013-22012/FFCD-9203/GERCOR phase II study. J Clin Oncol 2010; 28: 4450-4456.

23. Bilimoria KY, Bentrem DJ, Ko CY et al. Multimodality therapy for pancreatic cancer in the U.S. : utilization, outcomes, and the effect of hospital volume. Cancer 2007; 110: 1227-1234.

24. Boeck S, Ankerst DP, Heinemann V. The role of adjuvant chemotherapy for patients with resected pancreatic cancer: systematic review of randomized controlled trials and meta-analysis. Oncology 2007; 72: 314-321.

25. Winter JM, Cameron JL, Campbell KA et al. 1423 pancreaticoduodenectomies for pancreatic cancer: A single-institution 
experience. J Gastrointest Surg 2006; 10: 1199-1210; discussion 1210-1211.

26. Klempnauer J, Ridder GJ, Piso P et al. Is liver resection in metastases of exocrine pancreatic carcinoma justified? Chirurg 1996; 67: 366-370.

27. Klein F, Puhl G, Guckelberger O et al. The impact of simultaneous liver resection for occult liver metastases of pancreatic adenocarcinoma. Gastroenterol Res Pract 2012; 2012: 939350.

28. Gleisner AL, Assumpcao L, Cameron JL et al. Is resection of periampullary or pancreatic adenocarcinoma with synchronous hepatic metastasis justified? Cancer 2007; 110: 2484-2492.

29. Takada T, Yasuda H, Amano H et al. Simultaneous hepatic resection with pancreato-duodenectomy for metastatic pancreatic head carcinoma: does it improve survival? Hepatogastroenterology 1997; 44: 567-573.

30. Park JB, Kim YH, Kim J et al. Radiofrequency ablation of liver metastasis in patients with locally controlled pancreatic ductal adenocarcinoma. J Vasc Interv Radiol 2012; 23: 635-641.

31. Janoray G, Chapet $S$, Ruffier-Loubière A et al. Robotic stereotactic body radiation therapy for tumors of the liver: radiation-induced liver disease, incidence and predictive factors. Cancer Radiother 2014; 18: 191-197.

32. Bretschneider T, Ricke J, Gebauer B et al. Image-guided highdose-rate brachytherapy of malignancies in various inner organs - technique, indications, and perspectives. J Contemp Brachytherapy 2016; 8: 251-261. 